\title{
Recognition of Reviewers
}

The Japan Society for Occupational Health expresses its sincere appreciation to the following volunteers for reviewing manuscripts for Environmental and Occupational Health Practice from October 1, 2020 to September 30, 2021.

Aoyama, Tomoko (Japan)

Chiba, Rie (Japan)

Ebara, Takeshi (Japan)

Endo, Yoko (Japan)

Fujino, Yoshihisa (Japan)

Hinoue, Mitsuo (Japan)

Ichiba, Masayoshi (Japan)

Ikeda, Hiroki (Japan)

Ikegami, Kazunori (Japan)

Inaba, Ryoichi (Japan)

Ishimaru, Tomohiro (Japan)

Izumi, Hiroyuki (Japan)

Jinnouchi, Hiroshige (Japan)

Kabe, Isamu (Japan)

\author{
Kageyama, Takayuki (Japan) \\ Kakamu, Takeyasu (Japan) \\ Kanamori, Satoru (Japan) \\ Kawashima, Masatoshi (Japan) \\ Kikuchi, Hiroshi (Japan) \\ Kim, Ilsung (Japan) \\ Kouchi, Makiko (Japan) \\ Kuwahara, Keisuke (Japan) \\ Mafune, Kosuke (Japan) \\ Nagata, Masako (Japan) \\ Nagata, Tomohisa (Japan) \\ Nakahara, Hirohiko (Japan) \\ Nishimura, Yoshito (United States) \\ Odagami, Kiminori (Japan)
}

\author{
Odagiri, Yuko (Japan) \\ Ogami, Akira (Japan) \\ Park, Sungjin (Korea (the Republic of)) \\ Sakurazawa, Hirofumi (Japan) \\ Sasaki, Natsu (Japan) \\ Sunakawa, Mitsuhiro (Japan) \\ Suwazono, Yasushi (Japan) \\ Tachi, Norihide (Japan) \\ Takeuchi, Akito (Japan) \\ Tomonaga, Taisuke (Japan) \\ Tsukahara, Teruomi (Japan) \\ Watanabe, Kazuhiro (Japan) \\ Watanabe, Sintaroo (Japan) \\ Yoshioka, Akira (Japan)
}

